\title{
Regional analysis of public sector employment
}

\section{SUMMARY}

This article presents updated analysis of public sector employment by region, with time series since 1999. The article uses the latest figures from public sector organisations for Scotland, Northern Ireland and the UK and the recently reweighted Labour Force Survey data to estimate the breakdown for English regions and Wales.
1 egional estimates of public sector employment (PSE) are used for policy-related purposes and have been produced by the Office for National Statistics (ONS) using the Labour Force Survey (LFS), in conjunction with PSE estimates based on returns from public sector organisations, since 2005. The most recent estimates were produced in 2007 and featured in the March 2007 edition of Economic \& Labour Market Review.

This article uses the most up-to-date PSE estimates alongside the recently reweighted LFS data to produce regional estimates for the period 1999 to 2007. The main findings from the analysis are:

- in 2007, all regions of the UK have an increased number of people working within the public sector, compared with the figure for 1999. This masks recent declines in the level of PSE; in the two years since 2005, many regions have seen levels of PSE fall (except Wales, West Midlands, London, Yorkshire and The Humber and Northern Ireland)

- over the year to Q4 2007, the largest relative decrease in the number of people working in the public sector was in the East Midlands region (1.1 per cent), while the largest relative increase was in the West Midlands region ( 0.7 per cent)

- Northern Ireland continues to have the highest proportion of their workforce working within the public sector in the 12 months to Q4 2007 (28.7 per cent), followed by Wales (23.6 per cent), the North East (22.9 per cent) and Scotland
(22.5 per cent). For the same time period, the East Midlands (17.0 per cent), East (17.1 per cent), South East (17.3 per cent) and London (18.5 per cent) had the smallest proportion of their workforce working in the public sector

\section{Regional public sector employment estimates}

Estimates of PSE are produced for Scotland and Northern Ireland using returns from public sector organisations and are compiled by the Scottish Government and the Department of Enterprise, Trade and Investment for Northern Ireland (DETINI), respectively. These 'administrative-based' estimates are in fact based on a combination of administrative data and estimates from surveys of public sector organisations. For the purpose of this article, these estimates will be referred to as administrative-based, since most of the surveys approach 100 per cent in coverage. UK PSE estimates are produced at ONS and combine the Scottish and Northern Irish administrative-based figures with administrative-based estimates for England and Wales combined. Separate PSE estimates for England and Wales are not available from administrative sources and therefore estimates have been provided periodically by combining administrative data with data from the LFS.

The most recent publication of regional PSE estimates was in 2007. Since then, the LFS microdata has been subject to a major reweighting exercise, to take account of changes in population profiles at a local level that have occurred since the 
last reweighting exercise in 2003. More information regarding the 2007-weighted LFS microdata can be found in Hughes and Palmer (2008).

\section{Using the LFS for regional estimates of PSE}

The LFS is a survey of households and among other things collects information regarding the sector of employment and region of workplace. It is therefore possible to produce regional breakdowns of PSE using the LFS. However, as highlighted in Millard (2007), the LFS tends to overestimate PSE, relative to the administrative PSE series, for a number of reasons:

- public/private and industry classification rely on the respondent's view of the organisation they work for, whereas administrative estimates use information directly from the organisations. The respondent-based approach can lead to reporting error.
Analysis has highlighted that people sometimes associate their employer by their place of work, rather than the organisation that pays their wage. For example, a person working as a catering assistant in a school might state that they work for a school, even if their wage is paid by a private catering firm

- the LFS public/private variable (PUBLICR) does not fully match the National Accounts definition of the public sector used to produce the administrative PSE estimates. In particular, university staff and GPs should be classified under the private sector according to National Accounts definitions, whereas in the LFS they are both classified as belonging to the public sector

- the administrative PSE survey method tends to lead to undercoverage of schools devolved from local government

\section{Figure 1}

\section{Comparison of PSE estimates from public sector organisations} and LFS

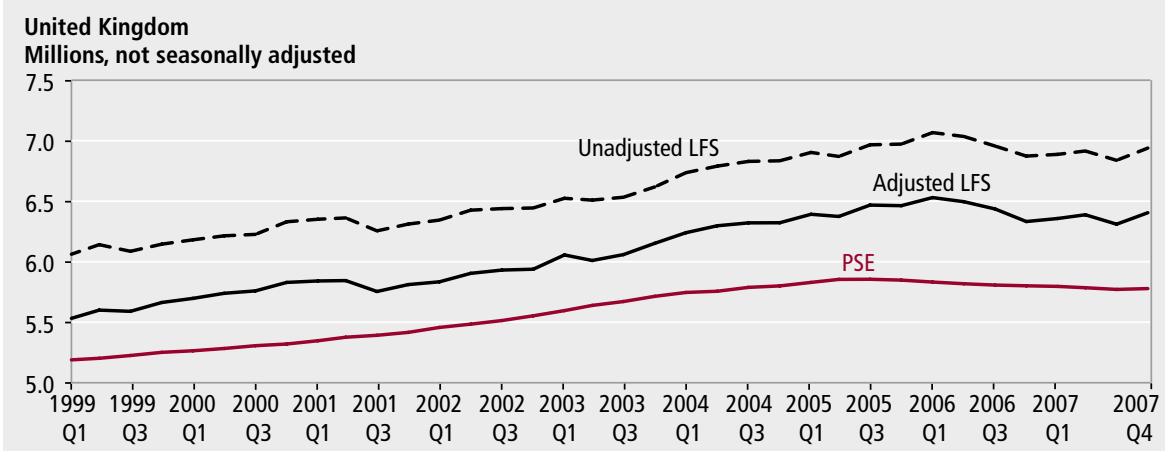

Source: Labour Force Survey; returns from public sector organisations (ONS, Scottish Government and Department of Enterprise, Trade and Investment for Northern Ireland)

Figure 2

Public sector employment as a proportion of all in employment: by region and country ${ }^{1}$ of workplace, year to Q4 $2007^{2}$

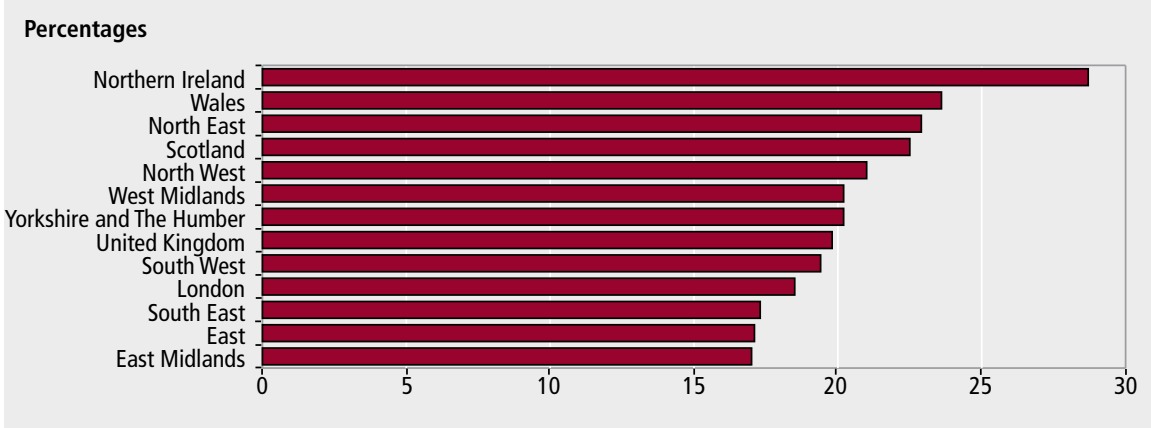

\section{Notes:}

1 Public sector statistics for Northern Ireland relate to the number of public sector jobs rather than the number of people working in the public sector. HM Forces figures are not included in Northern Ireland estimates.

2 Four-quarterly averages are based on estimates over the quarters March (Q1), June (Q2), September (Q3) and December (Q4) 2007.

Source: Labour Force Survey; returns from public sector organisations (ONS, Scottish Government and Department of Enterprise, Trade and Investment for Northern Ireland)

It is possible to adjust the LFS to bring the public sector variable closer to the National Accounts definition. Figure 1 compares the PSE administrative series with the LFS and 'adjusted' LFS figures and illustrates the size of the LFS overestimation. It is clear that, under the adjusted methodology, the divergence between the LFS and PSE estimates is approximately halved.

The method utilised by this article closely follows that undertaken in Millard (2007). Seasonally adjusted LFS estimates are presented to ensure consistency with the administrative-based UK PSE series. The LFS estimates are four-quarter rolling averages, to minimise any effects related to sampling variations. The estimates run from Q1 1999 to Q4 2007, in line with the availability of PSE estimates for Scotland.

Figures for Scotland and Northern Ireland are four-quarterly averages taken from the published PSE estimates. These are not seasonally adjusted. The figures quoted in this article will therefore differ from the estimates published by the Scottish Government and DETINI. For example, the data shown in this article for Scotland for Q4 2007 is an average taken for the quarters Q1 to Q4 2007, whereas the published Scottish Government estimate for Q4 2007 refers to employment as at December 2007. Additionally, estimates of rates of PSE published by DETINI are typically expressed relative to the total number of employee jobs rather than the LFS-based estimate of total employment, as quoted in this article.

The regional split for England, Wales and the English regions is generated by prorating the England and Wales administrative total (the administrativebased UK PSE figure minus the administrative Scotland and Northern Ireland PSE estimates) according to the adjusted LFS proportions. Further explanation is available in Millard (2007).

\section{Updated estimates}

For the four quarters to Q4 2007, the regions having the highest proportion of their workforce in the public sector continued to be Northern Ireland (28.7 per cent), Wales (23.6 per cent), the North East (22.9 per cent) and Scotland (22.5 per cent). The regions with the lowest proportion of their workforce in the public sector were the East Midlands (17.0 per cent), East (17.1 per cent), South East (17.3 per cent) and London (18.5 per cent). This is presented in Figure 2. The rates are similar to those presented in Millard (2007) for the four quarters to Q3 2006. 
Figure 3

Public sector employment as a proportion of total resident population: by region and country, ${ }^{1} 2006^{2,3}$

Percentages

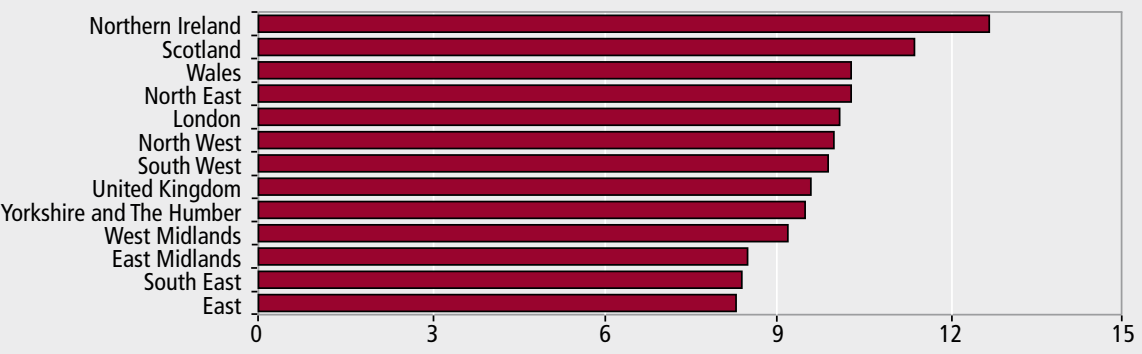

\section{Notes:}

1 Public sector statistics for Northern Ireland relate to the number of public sector jobs rather than the number of people working in the public sector. HM Forces figures are not included in Northern Ireland estimates.

2 Headcount, four-quarterly averages are based on estimates over the quarters March (Q1), June (Q2), September (Q3) and December (Q4) 2006.

3 Public sector employment estimates are workplace-based estimates, that is, where people work rather than where they live. Mid-year population estimates measure resident population.

Source: Labour Force Survey; returns from public sector organisations (ONS, Scottish Government and Department of Enterprise, Trade and Investment for Northern Ireland); ONS mid-year 2006 population estimates

Table 1

Public sector employment: ${ }^{1}$ by region and country of workplace
It is also possible to express PSE as a proportion of the resident population, illustrating the relationship between the size of the public sector and the size of the population it serves. Variation between regions is smaller - for the four quarters to Q4 2006, the range is between 8.3 per cent for the East and 12.7 per cent for Northern Ireland. This is presented in Figure 3.

Again, the proportions are similar to those presented in Millard (2007).

Between 1999 and 2007, all regions increased the level of PSE (see Table 1 and Table 2). Over the period 1999 to 2007, the region with the largest relative increase in the number of people working in the public sector was the West Midlands, which had a 17.8 per cent rise. London was the region with the smallest rise, showing an increase of 6.5 per cent.

Between Q4 2005 and Q4 2007, all

\begin{tabular}{|c|c|c|c|c|c|c|c|c|c|c|c|c|c|c|c|}
\hline \multicolumn{16}{|c|}{ Thousands, seasonally adjusted } \\
\hline $\begin{array}{l}\text { Average } \\
\text { of four } \\
\text { quarters to: }\end{array}$ & $\begin{array}{l}\text { North } \\
:{ }^{2} \text { East }\end{array}$ & $\begin{array}{l}\text { North } \\
\text { West }\end{array}$ & $\begin{array}{c}\text { Yorkshire } \\
\text { and The } \\
\text { Humber }\end{array}$ & $\begin{array}{r}\text { East } \\
\text { Midlands }\end{array}$ & $\begin{array}{r}\text { West } \\
\text { Midlands }\end{array}$ & East & London & $\begin{array}{r}\text { South } \\
\text { East }\end{array}$ & $\begin{array}{c}\text { South } \\
\text { West }\end{array}$ & England & Wales & Scotland $^{3}$ & $\begin{array}{l}\text { Great } \\
\text { Britain }\end{array}$ & $\begin{array}{r}\text { Northern } \\
\text { Ireland }^{4}\end{array}$ & $\begin{array}{r}\text { United } \\
\text { Kingdom }\end{array}$ \\
\hline \multicolumn{16}{|l|}{ PSE level } \\
\hline 1999Q4 & 240 & 591 & 438 & 318 & 430 & 411 & 716 & 626 & 429 & 4,200 & 290 & 529 & 5,019 & 197 & 5,217 \\
\hline $2000 Q 1$ & 238 & 597 & 439 & 319 & 437 & 415 & 719 & 629 & 426 & 4,219 & 289 & 530 & 5,037 & 198 & 5,236 \\
\hline 2000Q2 & 235 & 609 & 444 & 320 & 439 & 417 & 715 & 629 & 430 & 4,238 & 288 & 531 & 5,057 & 199 & 5,256 \\
\hline 2000Q3 & 232 & 621 & 452 & 318 & 434 & 421 & 703 & 635 & 438 & 4,254 & 291 & 532 & 5,077 & 199 & 5,276 \\
\hline 2000Q4 & 230 & 633 & 456 & 315 & 430 & 426 & 691 & 640 & 446 & 4,267 & 295 & 532 & 5,094 & 200 & 5,293 \\
\hline $2001 Q 1$ & 232 & 643 & 459 & 315 & 429 & 429 & 683 & 640 & 453 & 4,283 & 299 & 532 & 5,114 & 200 & 5,314 \\
\hline 2001Q2 & 234 & 650 & 458 & 314 & 431 & 431 & 687 & 643 & 455 & 4,303 & 300 & 533 & 5,136 & 201 & 5,337 \\
\hline 2001Q3 & 238 & 654 & 449 & 317 & 439 & 431 & 703 & 640 & 454 & 4,324 & 299 & 534 & 5,157 & 202 & 5,359 \\
\hline $2001 Q 4$ & 245 & 661 & 445 & 320 & 445 & 431 & 711 & 638 & 454 & 4,349 & 296 & 536 & 5,180 & 202 & 5,383 \\
\hline 2002Q1 & 249 & 666 & 440 & 320 & 451 & 429 & 723 & 642 & 457 & 4,377 & 293 & 538 & 5,207 & 203 & 5,410 \\
\hline 2002Q2 & 251 & 665 & 440 & 324 & 457 & 431 & 726 & 644 & 462 & 4,400 & 293 & 540 & 5,234 & 204 & 5,437 \\
\hline $2002 Q 3$ & 256 & 665 & 448 & 329 & 457 & 430 & 724 & 647 & 466 & 4,423 & 297 & 543 & 5,263 & 205 & 5,468 \\
\hline 2002Q4 & 256 & 666 & 455 & 331 & 462 & 435 & 728 & 649 & 468 & 4,450 & 300 & 545 & 5,296 & 206 & 5,502 \\
\hline 2003Q1 & 253 & 666 & 464 & 331 & 468 & 441 & 727 & 649 & 476 & 4,475 & 306 & 548 & 5,329 & 208 & 5,537 \\
\hline 2003Q2 & 255 & 673 & 472 & 332 & 469 & 447 & 723 & 650 & 482 & 4,503 & 312 & 551 & 5,366 & 209 & 5,576 \\
\hline $2003 Q 3$ & 255 & 678 & 480 & 333 & 472 & 455 & 722 & 653 & 484 & 4,532 & 318 & 554 & 5,404 & 211 & 5,615 \\
\hline $2003 Q 4$ & 251 & 678 & 490 & 338 & 471 & 465 & 723 & 662 & 488 & 4,566 & 319 & 558 & 5,443 & 212 & 5,655 \\
\hline $2004 Q 1$ & 252 & 684 & 502 & 350 & 468 & 474 & 721 & 668 & 481 & 4,599 & 319 & 561 & 5,479 & 213 & 5,693 \\
\hline 2004Q2 & 254 & 681 & 511 & 359 & 466 & 481 & 726 & 678 & 477 & 4,631 & 312 & 565 & 5,508 & 214 & 5,722 \\
\hline $2004 Q 3$ & 256 & 682 & 511 & 367 & 468 & 488 & 731 & 683 & 477 & 4,663 & 305 & 568 & 5,536 & 215 & 5,751 \\
\hline 2004Q4 & 261 & 684 & 508 & 371 & 473 & 487 & 734 & 686 & 477 & 4,681 & 304 & 571 & 5,556 & 216 & 5,773 \\
\hline $2005 Q 1$ & 269 & 685 & 500 & 365 & 476 & 485 & 743 & 691 & 488 & 4,701 & 301 & 574 & 5,576 & 217 & 5,793 \\
\hline 2005Q2 & 268 & 689 & 492 & 359 & 486 & 484 & 746 & 696 & 500 & 4,720 & 303 & 576 & 5,599 & 219 & 5,818 \\
\hline $2005 Q 3$ & 267 & 692 & 490 & 356 & 492 & 479 & 749 & 701 & 508 & 4,733 & 303 & 579 & 5,615 & 220 & 5,835 \\
\hline 2005Q4 & 264 & 689 & 492 & 358 & 493 & 476 & 754 & 703 & 514 & 4,744 & 302 & 581 & 5,626 & 220 & 5,847 \\
\hline 2006Q1 & 262 & 683 & 496 & 366 & 495 & 470 & 755 & 698 & 514 & 4,739 & 305 & 583 & 5,627 & 220 & 5,847 \\
\hline 2006Q2 & 263 & 679 & 494 & 373 & 494 & 463 & 762 & 691 & 510 & 4,729 & 304 & 584 & 5,618 & 220 & 5,838 \\
\hline 2006Q3 & 265 & 674 & 487 & 375 & 492 & 461 & 765 & 686 & 509 & 4,713 & 308 & 584 & 5,605 & 221 & 5,826 \\
\hline 2006Q4 & 266 & 680 & 482 & 371 & 491 & 457 & 765 & 679 & 507 & 4,699 & 310 & 584 & 5,593 & 221 & 5,815 \\
\hline 2007Q1 & 265 & 686 & 477 & 364 & 490 & 458 & 771 & 676 & 504 & 4,691 & 311 & 583 & 5,584 & 221 & 5,806 \\
\hline 2007Q2 & 264 & 686 & 474 & 356 & 494 & 454 & 771 & 680 & 500 & 4,680 & 315 & 581 & 5,576 & 221 & 5,797 \\
\hline 2007Q3 & 263 & 680 & 483 & 349 & 499 & 447 & 771 & 682 & 496 & 4,672 & 316 & 579 & 5,568 & 221 & 5,788 \\
\hline 2007Q4 & 264 & 673 & 493 & 346 & 507 & 443 & 763 & 688 & 495 & 4,670 & 314 & 578 & 5,562 & 220 & 5,783 \\
\hline
\end{tabular}

Change 2005 Q4 to $2007 Q 4$

$0 \quad-16$
Percentage change 2005Q4 to 2007Q4

$$
\begin{array}{lll}
-0.2 & -2.3 & 0.1
\end{array}
$$

$0.1-3.3$ 
Table 1 continued

\begin{tabular}{|c|c|c|c|c|c|c|c|c|c|c|c|c|c|c|c|}
\hline \multicolumn{2}{|c|}{$\begin{array}{l}\begin{array}{l}\text { Average } \\
\text { of four North } \\
\text { quarters to: }{ }^{2} \text { East }\end{array} \\
\text { Change on year }\end{array}$} & $\begin{array}{l}\text { North } \\
\text { West }\end{array}$ & $\begin{array}{l}\text { Yorkshire } \\
\text { and The } \\
\text { Humber }\end{array}$ & $\begin{array}{r}\text { East } \\
\text { Midlands }\end{array}$ & $\begin{array}{r}\text { West } \\
\text { Midlands }\end{array}$ & East & London & $\begin{array}{r}\text { South } \\
\text { East }\end{array}$ & $\begin{array}{r}\text { South } \\
\text { West }\end{array}$ & England & Wales & Scotland ${ }^{3}$ & $\begin{array}{r}\text { Great } \\
\text { Britain }\end{array}$ & $\begin{array}{l}\text { Northern } \\
\text { Ireland }{ }^{4} \mathrm{~K}\end{array}$ & $\begin{array}{l}\text { United } \\
\text { Singdom }\end{array}$ \\
\hline \\
\hline $2000 \mathrm{Q} 4$ & -9 & 41 & 18 & -3 & 0 & 15 & -26 & 14 & 17 & 67 & 4 & 3 & 74 & 2 & 77 \\
\hline 2001Q1 & -5 & 46 & 20 & -4 & -8 & 14 & -36 & 11 & 26 & 64 & 10 & 2 & 76 & 2 & 79 \\
\hline 2001Q2 & -1 & 41 & 13 & -6 & -8 & 13 & -28 & 14 & 26 & 65 & 12 & 2 & 79 & 2 & 82 \\
\hline $2001 Q 3$ & 6 & 33 & -3 & -1 & 4 & 11 & 0 & 5 & 16 & 71 & 7 & 2 & 80 & 3 & 83 \\
\hline $2001 Q 4$ & 14 & 28 & -11 & 4 & 15 & 5 & 21 & -2 & 7 & 82 & 1 & 4 & 87 & 3 & 90 \\
\hline $2002 Q 1$ & 17 & 23 & -19 & 5 & 22 & 0 & 40 & 2 & 4 & 94 & -6 & 6 & 94 & 3 & 96 \\
\hline $2002 Q 2$ & 17 & 15 & -18 & 10 & 26 & 0 & 39 & 2 & 6 & 97 & -7 & 7 & 97 & 3 & 100 \\
\hline $2002 Q 3$ & 18 & 11 & 0 & 12 & 19 & -2 & 21 & 7 & 12 & 99 & -2 & 9 & 106 & 3 & 109 \\
\hline $2002 Q 4$ & 11 & 5 & 10 & 11 & 17 & 4 & 17 & 12 & 14 & 102 & 4 & 9 & 115 & 4 & 119 \\
\hline $2003 Q 1$ & 5 & 0 & 24 & 11 & 17 & 12 & 4 & 7 & 18 & 99 & 13 & 10 & 122 & 5 & 127 \\
\hline $2003 Q 2$ & 4 & 8 & 33 & 8 & 12 & 16 & -3 & 6 & 20 & 103 & 19 & 11 & 133 & 6 & 138 \\
\hline $2003 Q 3$ & -2 & 12 & 32 & 4 & 15 & 25 & -2 & 6 & 18 & 109 & 21 & 11 & 141 & 6 & 147 \\
\hline $2003 Q 4$ & -5 & 12 & 35 & 7 & 9 & 31 & -6 & 13 & 20 & 116 & 19 & 13 & 147 & 6 & 153 \\
\hline $2004 Q 1$ & -2 & 17 & 38 & 18 & -1 & 33 & -6 & 19 & 6 & 123 & 13 & 14 & 150 & 6 & 156 \\
\hline $2004 Q 2$ & -2 & 8 & 39 & 27 & -3 & 34 & 3 & 28 & -6 & 128 & 0 & 14 & 142 & 5 & 146 \\
\hline $2004 Q 3$ & 1 & 4 & 30 & 34 & -4 & 34 & 9 & 30 & -7 & 131 & -13 & 14 & 132 & 4 & 136 \\
\hline $2004 Q 4$ & 10 & 6 & 19 & 32 & 2 & 21 & 12 & 24 & -11 & 115 & -15 & 14 & 113 & 4 & 118 \\
\hline $2005 Q 1$ & 17 & 1 & -2 & 15 & 9 & 11 & 22 & 23 & 6 & 102 & -18 & 13 & 97 & 4 & 101 \\
\hline 2005Q2 & 15 & 8 & -19 & 0 & 20 & 4 & 20 & 18 & 23 & 88 & -9 & 12 & 91 & 5 & 96 \\
\hline 200503 & 11 & 10 & -20 & -11 & 24 & -9 & 18 & 18 & 31 & 71 & -2 & 11 & 79 & 4 & 84 \\
\hline $2005 Q 4$ & 3 & 5 & -16 & -13 & 20 & -10 & 19 & 17 & 38 & 62 & -2 & 9 & 70 & 4 & 74 \\
\hline $2006 Q 1$ & -6 & -2 & -4 & 1 & 19 & -15 & 12 & 8 & 26 & 38 & 3 & 9 & 51 & 3 & 54 \\
\hline $2006 Q 2$ & -5 & -10 & 2 & 14 & 8 & -21 & 16 & -5 & 10 & 9 & 2 & 8 & 19 & 1 & 21 \\
\hline $2006 Q 3$ & -2 & -18 & -3 & 19 & 1 & -18 & 15 & -14 & 1 & -20 & 5 & 5 & -9 & 1 & -8 \\
\hline $2006 Q 4$ & 2 & -9 & -10 & 13 & -2 & -19 & 11 & -23 & -7 & -44 & 8 & 3 & -33 & 1 & -32 \\
\hline $2007 Q 1$ & 3 & 3 & -19 & -1 & -5 & -12 & 16 & -22 & -10 & -48 & 6 & -1 & -43 & 1 & -42 \\
\hline $2007 Q 2$ & 1 & 7 & -20 & -16 & -1 & -9 & 9 & -11 & -10 & -49 & 11 & -3 & -42 & 1 & -41 \\
\hline $2007 Q 3$ & -1 & 7 & -4 & -26 & 7 & -14 & 7 & -4 & -12 & -41 & 8 & -5 & -38 & 0 & -38 \\
\hline $2007 Q 4$ & -2 & -7 & 10 & -25 & 16 & -15 & -2 & 8 & -12 & -29 & 4 & -5 & -31 & -1 & -32 \\
\hline
\end{tabular}

\section{Notes:}

1 Headcount; rolling four-quarter averages.

2 Rolling four-quarterly averages are based on estimates over the quarters March (Q1), June (Q2), September (Q3) and December (Q4). For example, the Q4 1999 estimate is an average taken for the quarters Q1 1999 to Q4 1999.

3 Public sector employment estimates for Scotland are published by Scottish Government on a quarterly basis back to Q1 1999 from administrative records and surveys of public sector organisations in Scotland.

4 Public sector statistics for Northern Ireland relate to the number of public sector jobs rather than the number of people working in the public sector. The percentages for Northern Ireland as a proportion of all employment will differ from DETINI estimates expressed as a proportion of all jobs. HM Forces figures are not included in Northern Ireland estimates.

Source: Labour Force Survey; returns from public sector organisations (ONS, Scottish Government and Department of Enterprise, Trade and Investment for Northern Ireland)

regions have seen a decrease in the level of PSE, except Wales (3.9 per cent growth), the West Midlands (2.7 per cent), London (1.2 per cent), Yorkshire and The Humber and Northern Ireland (both 0.1 per cent). The region with the largest fall over this period was the East, in which the level fell by 7.0 per cent. Overall, the level of PSE in the UK between 2005 and 2007 fell by 1.1 per cent.

\section{Assessing the accuracy of ONS regional estimates of PSE}

Although estimates for Scotland have been taken from those published by the Scottish Government, it is possible to produce figures for Scotland based on the LFS methodology. The regional estimates can then be evaluated by comparing the two, as explained further in Millard (2007). Millard concluded that the differences between the two sources were no larger than the size that might be expected due to LFS sampling variability and respondent error.

A comparison of the two estimates is shown in Table 3. The variation between the two sources was of a very similar magnitude, as presented in Millard, at most 3.3 per cent more (Q3 2003) and 1.9 per cent less (Q4 1999).

\section{PSE development programme}

ONS is currently working with other government departments to develop regional PSE statistics from administrative sources, covering Northern Ireland,
Scotland, Wales and England (including English regional composition). This will incorporate existing regional administrative-based sources. Subject to a successful pilot study, the aim for future regional PSE estimates will be to replace the composite approach with the new administrative-based estimates.

\section{CONTACT}

elmr@ons.gsi.gov.uk 
Table 2

Public sector employment rate: by region and country of workplace ${ }^{1}$

Thousands, seasonally adjusted

\begin{tabular}{|c|c|c|c|c|c|c|c|c|c|c|c|c|c|c|c|}
\hline $\begin{array}{l}\text { Average } \\
\text { of four } \\
\text { quarters to }\end{array}$ & $\begin{array}{r}\text { North } \\
0:{ }^{2} \text { East }\end{array}$ & $\begin{array}{l}\text { North } \\
\text { West }\end{array}$ & $\begin{array}{c}\text { Yorkshire } \\
\text { and The } \\
\text { Humber }\end{array}$ & $\begin{array}{r}\text { East } \\
\text { Midlands }\end{array}$ & $\begin{array}{r}\text { West } \\
\text { Midlands }\end{array}$ & East & London & $\begin{array}{r}\text { South } \\
\text { East }\end{array}$ & $\begin{array}{c}\text { South } \\
\text { West }\end{array}$ & England & Wales & Scotland ${ }^{3}$ & $\begin{array}{r}\text { Great } \\
\text { Britain }\end{array}$ & $\begin{array}{l}\text { Northern } \\
\text { Ireland }{ }^{4} \mathrm{Ki}\end{array}$ & $\begin{array}{l}\text { United } \\
\text { ingdom }\end{array}$ \\
\hline \multicolumn{16}{|l|}{ PSE rate } \\
\hline 1999Q4 & 23.4 & 19.6 & 19.7 & 16.9 & 17.8 & 17.2 & 18.4 & 16.5 & 18.2 & 18.3 & 24.2 & 23.2 & 19.0 & 28.8 & 19.2 \\
\hline 2000Q1 & 23.0 & 19.6 & 19.8 & 16.9 & 18.1 & 17.3 & 18.4 & 16.5 & 18.1 & 18.3 & 24.0 & 23.1 & 19.0 & 28.9 & 19.2 \\
\hline 2000Q2 & 22.5 & 19.9 & 19.9 & 16.8 & 18.2 & 17.3 & 18.4 & 16.4 & 18.2 & 18.3 & 23.8 & 23.0 & 19.0 & 29.0 & 19.2 \\
\hline 2000Q3 & 22.1 & 20.3 & 20.2 & 16.7 & 18.1 & 17.2 & 18.1 & 16.5 & 18.4 & 18.3 & 24.0 & 22.9 & 19.0 & 29.1 & 19.2 \\
\hline 2000Q4 & 22.0 & 20.6 & 20.3 & 16.6 & 17.8 & 17.3 & 17.8 & 16.6 & 18.7 & 18.3 & 24.2 & 22.6 & 19.0 & 29.0 & 19.2 \\
\hline 2001Q1 & 22.2 & 20.9 & 20.4 & 16.6 & 17.8 & 17.2 & 17.6 & 16.5 & 18.9 & 18.4 & 24.5 & 22.6 & 19.0 & 29.0 & 19.3 \\
\hline 2001Q2 & 22.6 & 21.1 & 20.4 & 16.6 & 17.8 & 17.3 & 17.6 & 16.6 & 19.0 & 18.4 & 24.8 & 22.5 & 19.1 & 28.9 & 19.3 \\
\hline $2001 Q 3$ & 23.0 & 21.3 & 19.9 & 16.7 & 18.1 & 17.3 & 17.8 & 16.5 & 18.9 & 18.5 & 24.8 & 22.6 & 19.1 & 28.8 & 19.4 \\
\hline 2001Q4 & 23.6 & 21.5 & 19.8 & 16.9 & 18.2 & 17.3 & 17.9 & 16.4 & 18.8 & 18.5 & 24.7 & 22.7 & 19.2 & 29.0 & 19.4 \\
\hline $2002 Q 1$ & 23.8 & 21.6 & 19.4 & 16.8 & 18.4 & 17.2 & 18.2 & 16.5 & 18.9 & 18.6 & 24.5 & 22.8 & 19.2 & 29.2 & 19.5 \\
\hline 2002Q2 & 24.0 & 21.7 & 19.3 & 17.0 & 18.6 & 17.2 & 18.3 & 16.6 & 19.1 & 18.7 & 24.4 & 23.0 & 19.3 & 29.2 & 19.6 \\
\hline $2002 Q 3$ & 24.3 & 21.6 & 19.6 & 17.1 & 18.5 & 17.0 & 18.3 & 16.7 & 19.2 & 18.7 & 24.6 & 23.0 & 19.4 & 29.4 & 19.6 \\
\hline $2002 Q 4$ & 24.3 & 21.6 & 19.7 & 17.1 & 18.7 & 17.2 & 18.4 & 16.7 & 19.3 & 18.8 & 24.6 & 23.1 & 19.4 & 29.2 & 19.7 \\
\hline 2003Q1 & 24.0 & 21.5 & 20.1 & 17.1 & 18.9 & 17.5 & 18.5 & 16.7 & 19.5 & 18.9 & 24.7 & 23.1 & 19.5 & 28.8 & 19.8 \\
\hline 2003Q2 & 24.1 & 21.6 & 20.4 & 17.0 & 18.9 & 17.7 & 18.5 & 16.7 & 19.7 & 19.0 & 24.7 & 23.1 & 19.6 & 28.9 & 19.8 \\
\hline $2003 Q 3$ & 23.9 & 21.6 & 20.6 & 17.0 & 19.2 & 18.0 & 18.4 & 16.8 & 19.7 & 19.1 & 24.9 & 23.1 & 19.7 & 29.1 & 19.9 \\
\hline $2003 Q 4$ & 23.4 & 21.5 & 20.9 & 17.3 & 19.2 & 18.2 & 18.5 & 17.0 & 19.8 & 19.2 & 24.7 & 23.1 & 19.8 & 29.4 & 20.0 \\
\hline $2004 Q 1$ & 23.1 & 21.6 & 21.4 & 17.8 & 19.1 & 18.5 & 18.4 & 17.1 & 19.5 & 19.3 & 24.6 & 23.2 & 19.9 & 30.0 & 20.1 \\
\hline 2004Q2 & 23.2 & 21.5 & 21.7 & 18.2 & 18.9 & 18.7 & 18.4 & 17.4 & 19.3 & 19.4 & 24.0 & 23.2 & 19.9 & 30.3 & 20.2 \\
\hline $2004 Q 3$ & 23.2 & 21.4 & 21.6 & 18.7 & 18.9 & 18.9 & 18.6 & 17.5 & 19.3 & 19.4 & 23.5 & 23.3 & 20.0 & 30.5 & 20.2 \\
\hline $2004 Q 4$ & 23.7 & 21.4 & 21.4 & 18.9 & 19.0 & 18.9 & 18.6 & 17.6 & 19.3 & 19.5 & 23.4 & 23.3 & 20.0 & 30.4 & 20.3 \\
\hline 2005Q1 & 24.3 & 21.4 & 21.0 & 18.5 & 19.0 & 18.8 & 18.8 & 17.6 & 19.7 & 19.5 & 23.2 & 23.3 & 20.0 & 30.2 & 20.3 \\
\hline 2005Q2 & 24.1 & 21.5 & 20.6 & 18.1 & 19.3 & 18.8 & 18.9 & 17.7 & 20.1 & 19.5 & 23.3 & 23.5 & 20.0 & 30.1 & 20.3 \\
\hline $2005 Q 3$ & 24.0 & 21.6 & 20.4 & 17.8 & 19.5 & 18.6 & 18.9 & 17.7 & 20.3 & 19.5 & 23.1 & 23.5 & 20.1 & 29.9 & 20.3 \\
\hline 2005Q4 & 23.8 & 21.5 & 20.4 & 17.7 & 19.6 & 18.5 & 19.0 & 17.7 & 20.6 & 19.5 & 23.0 & 23.6 & 20.1 & 29.9 & 20.3 \\
\hline $2006 Q 1$ & 23.5 & 21.3 & 20.4 & 17.9 & 19.8 & 18.3 & 18.9 & 17.5 & 20.5 & 19.5 & 23.2 & 23.7 & 20.0 & 29.8 & 20.3 \\
\hline 2006Q2 & 23.4 & 21.1 & 20.3 & 18.2 & 19.7 & 18.1 & 19.1 & 17.3 & 20.3 & 19.4 & 23.2 & 23.7 & 19.9 & 29.5 & 20.2 \\
\hline $2006 Q 3$ & 23.5 & 20.9 & 20.0 & 18.3 & 19.6 & 18.0 & 19.1 & 17.2 & 20.2 & 19.3 & 23.5 & 23.6 & 19.9 & 29.4 & 20.1 \\
\hline 2006 Q4 & 23.5 & 21.1 & 19.8 & 18.1 & 19.5 & 17.8 & 19.0 & 17.0 & 20.1 & 19.2 & 23.7 & 23.4 & 19.8 & 29.2 & 20.0 \\
\hline 2007Q1 & 23.4 & 21.3 & 19.6 & 17.8 & 19.5 & 17.7 & 19.0 & 17.0 & 19.9 & 19.2 & 23.7 & 23.2 & 19.7 & 29.0 & 20.0 \\
\hline 2007Q2 & 23.3 & 21.4 & 19.6 & 17.5 & 19.7 & 17.5 & 18.9 & 17.1 & 19.8 & 19.1 & 23.9 & 22.8 & 19.7 & 28.9 & 19.9 \\
\hline $2007 Q 3$ & 23.1 & 21.2 & 19.9 & 17.2 & 20.0 & 17.3 & 18.8 & 17.2 & 19.6 & 19.1 & 23.8 & 22.6 & 19.6 & 28.7 & 19.8 \\
\hline 2007Q4 & 22.9 & 21.0 & 20.2 & 17.0 & 20.2 & 17.1 & 18.5 & 17.3 & 19.4 & 19.0 & 23.6 & 22.5 & 19.5 & 28.7 & 19.8 \\
\hline
\end{tabular}

Notes:

1 Headcount; rolling four-quarter averages.

2 Rolling four-quarterly averages are based on estimates over the quarters March (Q1), June (Q2), September (Q3) and December (Q4). For example, the Q4 1999 estimate is an average taken for the quarters Q1 1999 to Q4 1999.

3 Public sector employment estimates for Scotland are published by Scottish Government on a quarterly basis back to Q1 1999 from administrative records and surveys of public sector organisations in Scotland.

4 Public sector statistics for Northern Ireland relate to the number of public sector jobs rather than the number of people working in the public sector. The percentages for Northern Ireland as a proportion of all employment will differ from DETINI estimates expressed as a proportion of all jobs. HM Forces figures are not included in Northern Ireland estimates.

Source: Labour Force Survey; returns from public sector organisations (ONS, Scottish Government and Department of Enterprise, Trade and Investment for Northern Ireland) 
Table 3

\section{Comparison between Scottish Government ${ }^{1}$ and ONS PSE estimates}

$\underline{\text { Scotland }}$

\begin{tabular}{|c|c|c|}
\hline & Difference: ONS minus $S$ & estimates of PSE \\
\hline Average of four quarters to: ${ }^{2}$ & PSE levels (thousands) & PSE levels (percentage difference) \\
\hline $1999 Q 4$ & -10 & -1.9 \\
\hline $2000 Q 1$ & -6 & -1.1 \\
\hline $2000 Q 2$ & 0 & 0.0 \\
\hline $2000 Q 3$ & 4 & 0.7 \\
\hline $2000 Q 4$ & 0 & 0.0 \\
\hline $2001 Q 1$ & 0 & 0.0 \\
\hline $2001 Q 2$ & 1 & 0.1 \\
\hline $2001 Q 3$ & 1 & 0.2 \\
\hline $2001 Q 4$ & 3 & 0.5 \\
\hline $2002 Q 1$ & 8 & 1.5 \\
\hline $2002 Q 2$ & 6 & 1.2 \\
\hline $2002 Q 3$ & 10 & 1.8 \\
\hline $2002 Q 4$ & 10 & 1.8 \\
\hline 2003Q1 & 11 & 2.0 \\
\hline $2003 Q 2$ & 12 & 2.1 \\
\hline $2003 Q 3$ & 18 & 3.3 \\
\hline $2003 Q 4$ & 11 & 2.0 \\
\hline $2004 Q 1$ & 9 & 1.5 \\
\hline 2004Q2 & 6 & 1.1 \\
\hline $2004 Q 3$ & 9 & 1.5 \\
\hline $2004 Q 4$ & 7 & 1.1 \\
\hline 2005Q1 & 12 & 2.0 \\
\hline 2005Q2 & 11 & 1.9 \\
\hline $2005 Q 3$ & 6 & 1.0 \\
\hline 2005Q4 & 1 & 0.2 \\
\hline 2006Q1 & -3 & -0.6 \\
\hline $2006 Q 2$ & -7 & -1.3 \\
\hline $2006 Q 3$ & -5 & -0.9 \\
\hline $2006 Q 4$ & -5 & -0.9 \\
\hline 2007Q1 & 0 & 0.0 \\
\hline 2007Q2 & 7 & 1.2 \\
\hline $2007 Q 3$ & 13 & 2.2 \\
\hline $2007 Q 4$ & 9 & 1.5 \\
\hline
\end{tabular}

Notes:

1 Public sector employment estimates for Scotland are published by the Scottish Government on a quarterly basis back to Q1 1999 from administrative records and surveys of individual public sector organisations in Scotland.

2 Rolling four-quarterly averages are based on estimates over the quarters March (Q1), June (Q2), September (Q3) and December (Q4). For example, the Q4 1999 estimate is an average taken for the quarters Q1 to Q4 1999.

Source: Labour Force Survey; returns from public sector organisations (ONS, Scottish Government)

\section{REFERENCES}

Hicks S, Walling A, Heap D and Livesey D (2005) 'Public Sector Employment Trends 2005', Office for National Statistics at www.statistics.gov.uk/cci/article.asp?id=1293 Hughes M and Palmer N (2008) 'Labour Force Survey: reweighting and seasonal adjustment review' at www.statistics.gov.uk/cci/article. asp?id=1992 Millard B (2007) 'Regional analysis of public sector employment', Economic \& Labour Market Review 1(3), pp 17-23 and at www.statistics.gov.uk/cci/article. $a s p ? i d=1738$ 